\title{
SÍNTESE DE INDANOS: UMA SELEÇÃO DE MÉTODOS GERAIS E EFICIENTES
}

Helena M. C. Ferraz*, Andrea M. Aguilar, Luiz F. Silva Jr.* e Marcus V. Craveiro

Instituto de Química, Universidade de São Paulo, CP 26077, 05513-970 São Paulo - SP

Recebido em 16/8/04; aceito em 1/10/04; publicado na web em 17/2/05

SYNTHESIS OF INDANS: A SELECTION OF GENERAL AND EFFICIENT METHODS. The indan ring system is present in several compounds with important pharmacological properties. In this account recent examples of selected methods (Friedel-Crafts acylation, cycloaddition reactions, ring contraction, cyclization and resolution) for the synthesis of indans are discussed.

Keywords: indan; Friedel-Crafts acylation; ring contraction.

\section{INTRODUÇÃO}

A unidade indânica consiste de um benzeno fundido a um anel ciclopentânico, podendo conter substituintes, tanto no anel aromático quanto no alifático (Figura 1). A rigidez e a planaridade são características importantes deste esqueleto, que está presente em diversas moléculas com atividade biológica de interesse, incluindo fármacos em comercialização, como o Indinavir ${ }^{\circledR}$ um inibidor da HIV-protease ${ }^{1,2}$ e o Aricept ${ }^{\circledR}$ utilizado no tratamento do mal de Alzheimer $^{3,4}$ (Figura 2).<smiles>c1ccc2c(c1)CCC2</smiles>

Figura 1. Esqueleto indânico
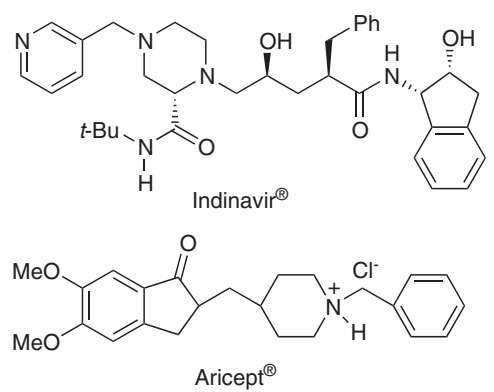

Figura 2. Exemplos de fármacos que possuem o esqueleto indânico

Vários derivados indânicos foram estudados em formulações de fragrância e demonstraram aumentar, melhorar ou modificar a eficácia das formulações em que foram incorporados ${ }^{5}$ (Figura 3).

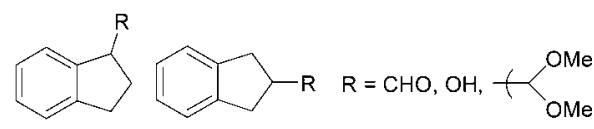

Figura 3. Exemplos de compostos utilizados em fragrâncias

*e-mail: hmferraz@iq.usp.br
Diante da importância dos compostos que possuem o esqueleto indânico, um grande número de métodos sintéticos tem sido desenvolvido nos últimos anos. Nos itens seguintes serão apresentados alguns trabalhos recentes sobre métodos gerais e eficientes de preparação de indanos, que foram publicados no período entre 1997-2004.

\section{ACILAÇÃO DE FRIEDEL-CRAFTS}

A reação de acilação de Friedel-Crafts intramolecular é, certamente, um dos métodos mais simples e eficientes para a síntese de indanonas funcionalizadas. O modo mais tradicional de realizar esta ciclização é o tratamento de um haleto de acila contendo um anel benzênico com um ácido de Lewis. Os haletos de acila, por sua vez, são normalmente preparados a partir do correspondente ácido carboxílico com cloreto de tionila. Utilizando este protocolo típico, algumas indanonas foram recentemente obtidas, como discutido nos três exemplos subseqüentes.

Uma seqüência de três etapas permitiu realizar a acilação do ácido $\beta$-fenil- $\alpha$-hidroxipropanóico, fornecendo a 2-acetóxi-1indanona, que é um intermediário na síntese diastereosseletiva do cis e do trans 1,2-indanodiol ${ }^{6}$ (Esquema 1).

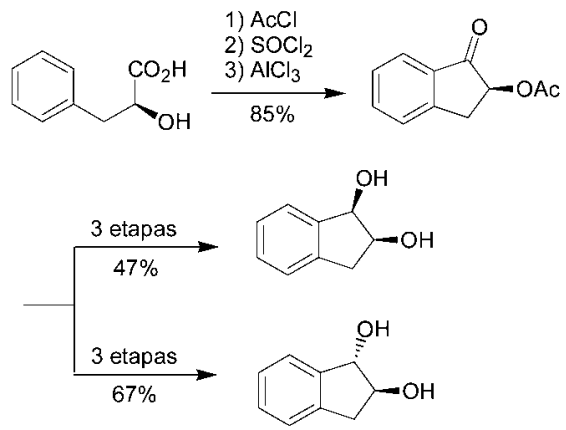

Esquema 1

As indanonas 2 também foram preparadas através da acilação de Friedel-Crafts. Neste procedimento, a conversão dos ácidos carboxílicos 1 aos cloretos de ácido correspondentes foi realizada com cloreto de oxalila. O cloreto obtido foi então tratado com cloreto de alumínio, fornecendo as indanonas $\mathbf{2}$. Transformações subseqüentes forneceram os indanóis $\mathbf{3}$ enantiomericamente pu$\operatorname{ros}^{7}$ (Esquema 2). 


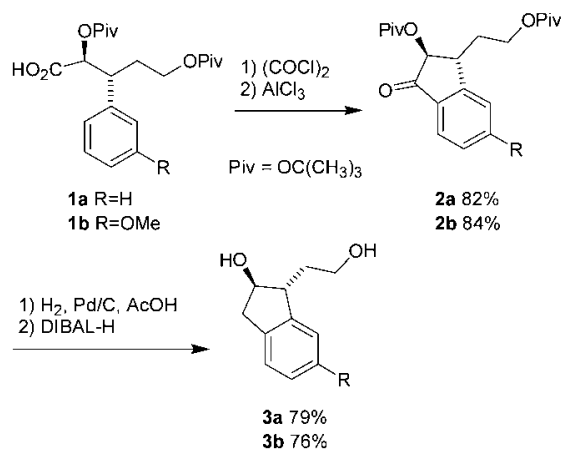

Esquema 2

A indanona $\mathbf{5}$ foi preparada através do tratamento com tetracloreto de titânio(IV) de um cloreto de acila, que foi preparado a partir do ácido 4 (Esquema 3). Quatro etapas subseqüentes permitiram transformar $\mathbf{5}$ na indanona $\mathbf{6}$, a qual foi utilizada como inibidor da luciferase da Renilla reniformis, em um estudo sobre bioluminescência de organismos marinhos ${ }^{8}$.

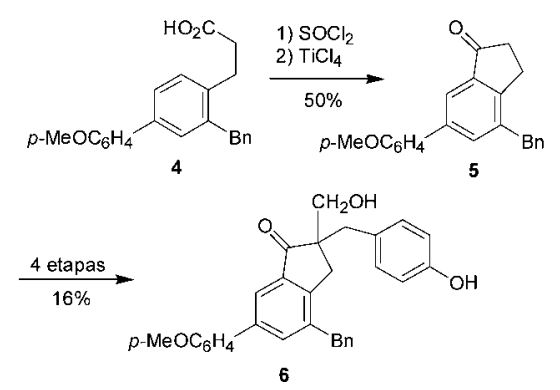

Esquema 3

Um outro procedimento muito utilizado para realizar uma acilação de Friedel-Crafts intramolecular é o tratamento de um ácido 3-arilpropiônico com ácidos próticos, evitando, assim, a etapa de formação do haleto de acila. Esta reação foi recentemente utilizada na síntese total de dois interessantes indanos: a (+)indatralina e o $( \pm)$-mutisiantol. A (+)-(1R,3S)-indatralina é uma droga sintética utilizada no tratamento de dependentes de drogas de abuso, como a cocaína ${ }^{9}$. Além disso, o enantiômero $(+)-(1 R, 3 S)$ mostrou-se 20 vezes mais potente que o enantiômero (-)-(1S,3R). A única síntese assimétrica da (+)-indatralina, realizada por Davies et $a l .{ }^{10}$, envolve uma acilação de Friedel-Crafts intramolecular mediada por ácido clorossulfônico do composto 7, para a formação da indanona intermediária $\mathbf{8}$, que após quatro etapas forneceu a (+)-indatralina (Esquema 4).<smiles>O=C(O)CC(c1ccccc1)c1ccc(Cl)c(Cl)c1</smiles><smiles>CN[C@H]1C[C@H](c2ccc(Cl)c(Cl)c2)c2ccccc21</smiles>

(+)-Indatralina

Esquema 4
Em 1997, Ho et al. ${ }^{11}$ publicaram uma síntese do sesquiterpeno fenólico mutisiantol. Nesta síntese, a acilação de Friedel-Crafts que forneceu a indanona $\mathbf{9}$ foi realizada com pentóxido de fósforo e ácido metanosulfônico (Esquema 5).

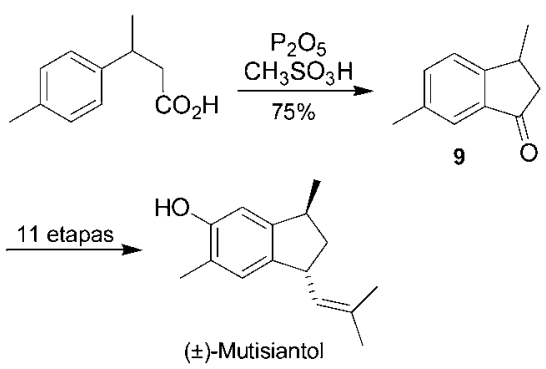

Esquema 5

Novos métodos têm sido desenvolvidos sobre a acilação de Friedel-Crafts, devido ao grande potencial desta reação na formação de compostos cíclicos, como indanonas. Uma série de 1indanonas substituídas no anel aromático foi preparada pelo tratamento de ácidos de Meldrum com quantidades catalíticas de triflato de escândio ${ }^{12}$ (Esquema 6). Um outro triflato de lantanídeo $\mathrm{Tb}(\mathrm{OTf})_{3}$ - também pode ser utilizado de maneira eficiente na síntese de indanonas. Neste caso, contudo, os substratos são ácidos $\operatorname{arilpropiônicos}^{13}$ (Esquema 7).<smiles>COc1cc2cc(OC)c1C1(C([O-])([O-])[O-])OC(C)(C)OC(=O)C1C2</smiles>

Esquema 6

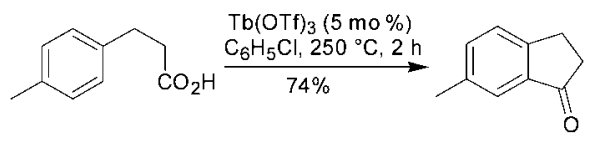

Esquema 7

\section{REAÇÕES DE CICLOADIÇÃO}

Dentre os métodos de preparação de indanos, as reações de cicloadição, juntamente com a acilação de Friedel-Crafts, estão entre as mais utilizadas.

A cicloadição formal [3+2] foi utilizada por Katritzky et al. ${ }^{14}$ para a preparação de indanos 1,3-dissubstituídos, através da reação de cátions benzílicos (10) com estirenos (Esquema 8).

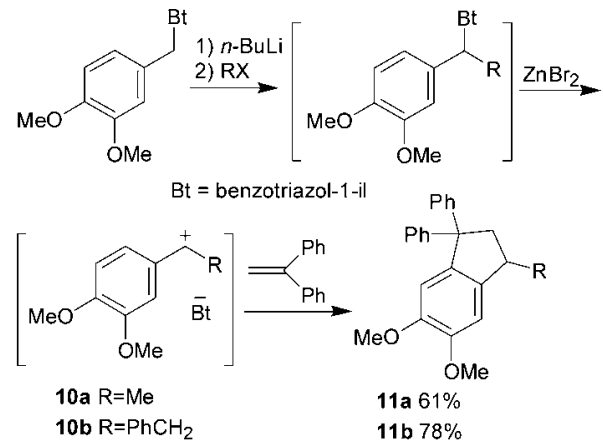


A cicloadição formal [3+2] intermolecular foi também utilizada na reação entre $p$-quinometanos, gerados in situ, e alquenos alifáticos ${ }^{15}$. A proposta mecanística para este procedimento envolve a oxidação do fenol 12 com DDQ, que deve fornecer o $p$-quinometano 15. O ataque nucleofílico do alqueno $\mathbf{1 3}$ ao $p$-quinometano $\mathbf{1 5}$ fornece o carbocátion terciário 16, que após uma alquilação de FriedelCrafts fornece o indano 17, em bom rendimento (Esquema 9).

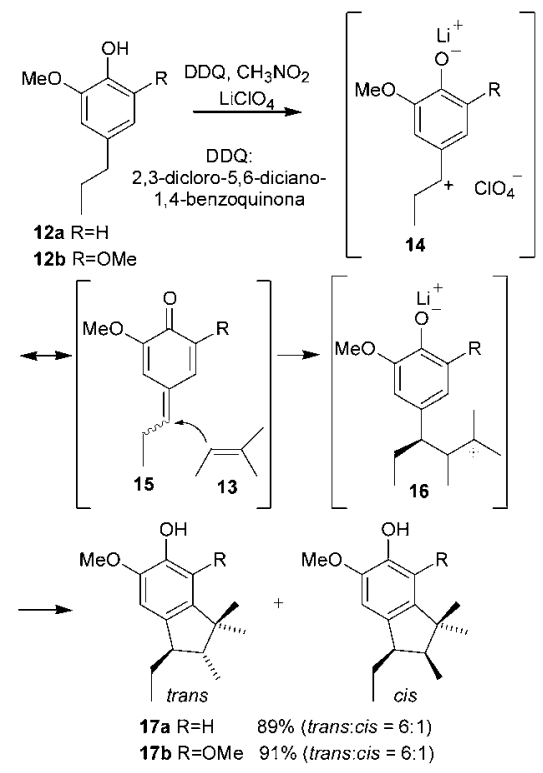

Esquema 9

Evidências para a reação de cicloadição intramolecular [2+4] entre um 1,3-diino e um alquino foram observadas por Johnson et $a l .{ }^{16}$. Utilizando este método foi possível preparar uma mistura do indano 18 e do indeno 19 (Esquema 10).

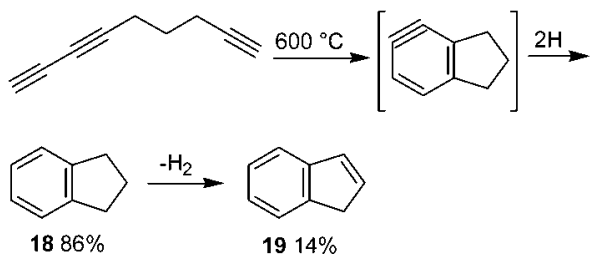

Esquema 10

Uma estratégia para a preparação de derivados de aminoácidos $\alpha, \alpha$-dissubstituídos que possuem o esqueleto indânico em sua estrutura utiliza a reação de Diels-Alder, seguida de uma aromatização, para fornecer o aminoácido $\mathbf{2 2}^{17}$ (Esquema 11). A preparação de aminoácidos que apresentam o esqueleto indânico foi descrita pelo grupo em $2000^{18}$, através da dialquilação do isocianoacetato de etila, utilizando $\mathrm{K}_{2} \mathrm{CO}_{3}$ como base e hidrogenossulfato de tetrabutilamônio,

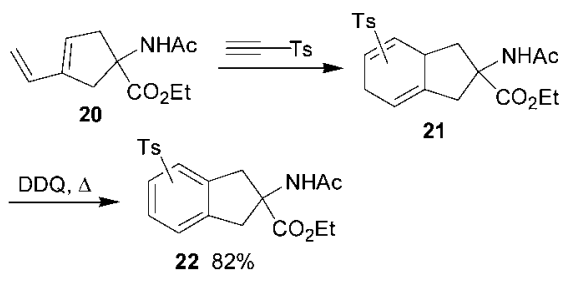

Esquema 11 como catalisador de transferência de fase (Esquema 12).

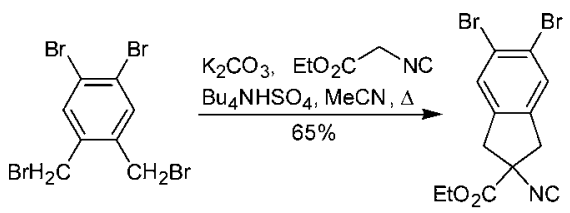

Esquema 12

A cicloadição catalisada por ródio do iodeto de arila $\mathbf{2 3}$, seguida de uma ciclização catalisada por paládio, foi realizada em uma única etapa por Grigg et al. ${ }^{19}$, fornecendo derivados indânicos funcionalizados (Esquema 13).
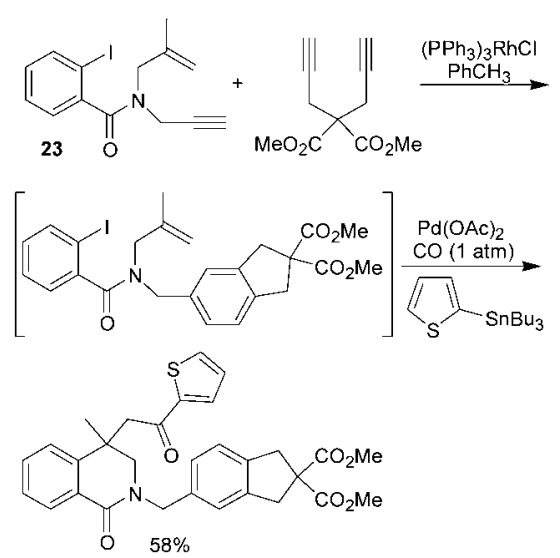

Esquema 13

Um outro procedimento descrito para a cicloadição intermolecular $[2+2+2]$ de alquinos foi desenvolvido utilizando paládio como catalisador ${ }^{20}$. A vantagem desta técnica é que foi possível usar quantidades quase equimolares do mono-alquino e do diino, uma vez que os procedimentos comuns empregam um grande excesso do mono-alquino para suprimir a dimerização do diino. Dessa maneira, foi possível obter o indano $\mathbf{2 6}$ através da formação de um paládio-ciclopentadieno, seguido da coordenação seletiva entre o paládio e o mono-alquino (Esquema 14).
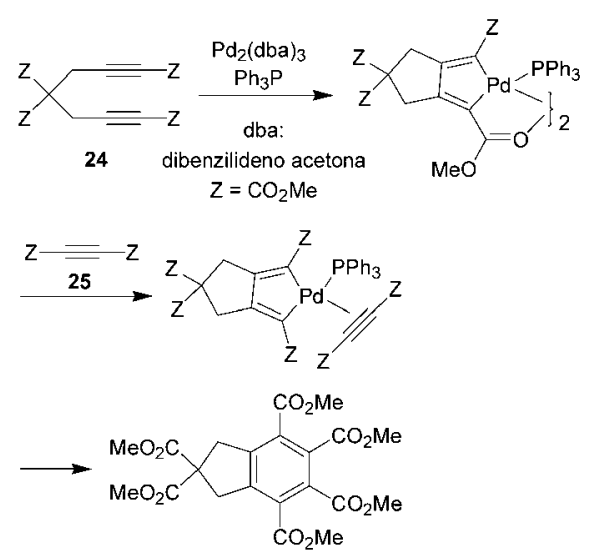

26 67\%

Esquema 14

Uma modificação interessante para a ciclotrimerização [2+2+2] foi descrita na preparação regiosseletiva de indanos, através da reação de cicloadição de diinos, simétricos e assimétricos, catalisada por níquel ${ }^{21}$ (Esquema 15). 


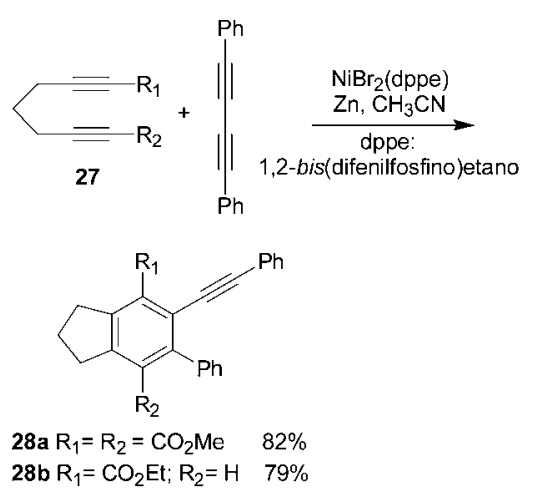

Esquema 15

Uma outra estratégia descrita para a síntese de 2-indanonas envolve a anelação [4+1] de sililarilcetenos com trimetilsilildiazometano $^{22}$. O substituinte de silício evita que ocorra a dimerização e a cicloadição [2+2] do arilceteno. O mecanismo proposto para esta anelação envolve a adição nucleofílica do silildiazometano ao ceteno 29, formando o respectivo enolato. O fechamento eletrocíclico deste enolato forma o derivado 30, que após isomerização fornece a indanona 31. O tratamento da indanona 31 com sílica gel fornece a monosililindanona 32 (Esquema 16).

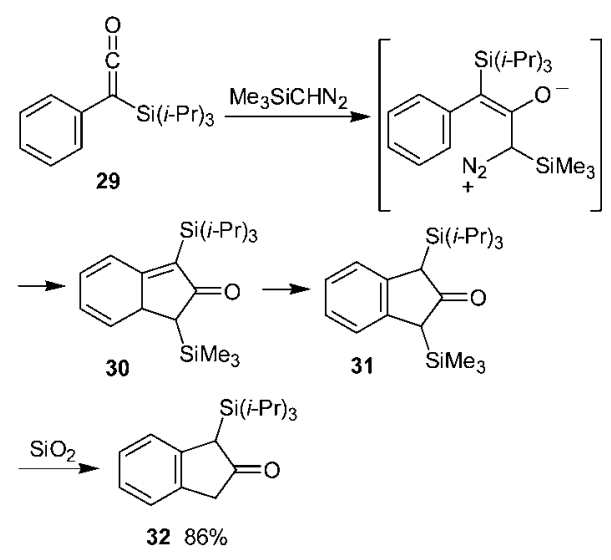

Esquema 16

Uma cicloadição [4+2] intramolecular catalisada por ouro entre um furano e um alquino foi utilizada na preparação de uma 3metil-1-indanona. Esta cetona foi transformada em duas etapas em uma mistura do sesquiterpeno natural jungianol e de seu epímero cis, sendo este último em maior proporção $0^{23}$ (Esquema 17).

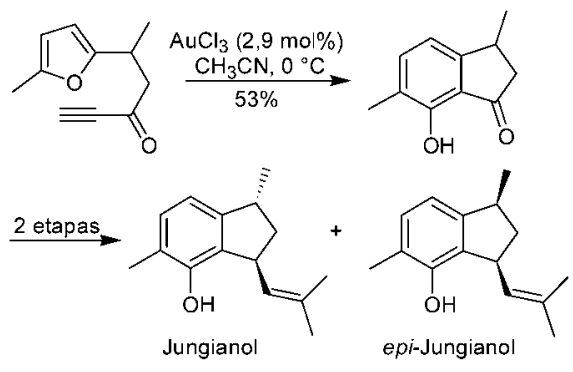

Esquema 17

Cicloadições também podem ser utilizadas na obtenção de indanos oticamente ativos, através do uso de um catalisador metálico quiral não-racêmico. Um exemplo recente desta abordagem foi a cicloadição $[2+2+2]$ de $\alpha, \omega$-diinos e monalquinos catalisada por um complexo de irídio, a qual permitiu a síntese de compostos axialmente quirais $^{24}$ (Esquema 18).

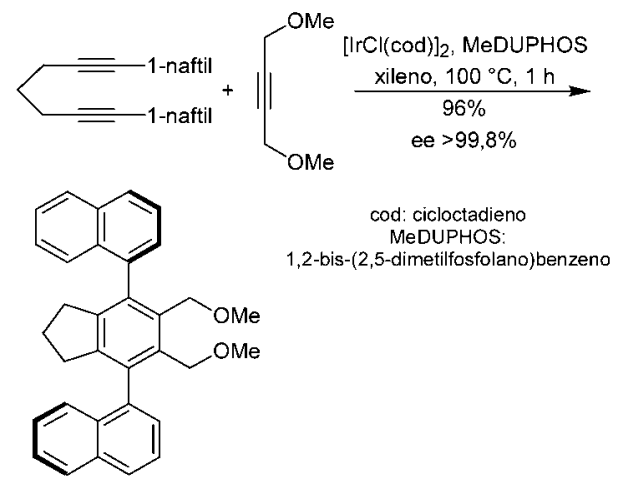

Esquema 18

\section{REAÇÕES DE CONTRAÇÃO DE ANEL}

O rearranjo oxidativo de moléculas contendo um carbociclo de seis membros pode ser uma estratégia interessante para a construção eficiente de indanos ${ }^{25}$. Como discutido a seguir, tálio(III) e iodo(III) são os dois oxidantes que foram utilizados com este propósito.

Diversos artigos descrevem a síntese de indanos através de uma contração de anel promovida por trinitrato de tálio (TTN). A primeira abordagem consiste da reação de 1-tetralonas com TTN adsorvido em $\mathrm{K}-10^{26}$. Neste caso, a contração não ocorreu com alguns dos substratos estudados e, quando aconteceu, os rendimentos obtidos foram apenas moderados.

A segunda abordagem foi pela reação de 1,2-diidronaftalenos com TTN, que forneceu indanos com bons rendimentos ${ }^{27}$ (Esquema 19 e Tabela 1). Cabe ressaltar que um único diastereoisômero foi obtido a partir do 4-metil-1,2-diidronaftaleno (Entrada 1, Tabela 1 e Esquema 20). A desvantagem deste método é que a reação de contração não ocorre com diidronaftalenos que possuem um grupo alquila na dupla ligação (Entrada 3). Entretanto, tal desvantagem pôde ser contornada utilizando-se 3-alquenóis análogos como substratos $^{28,29}$ (Tabela 2). Cabe ressaltar que também podem ser

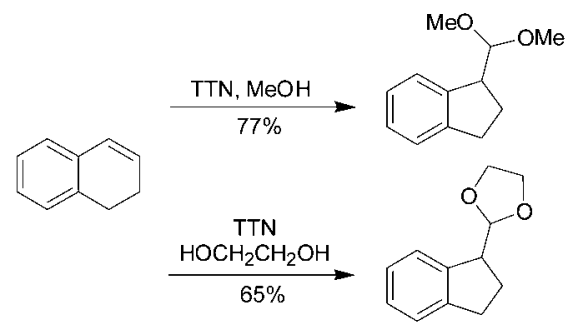

Esquema 19

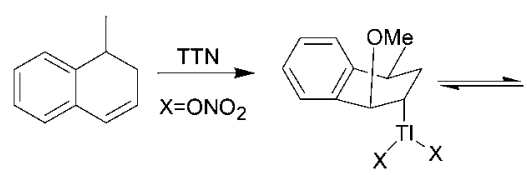<smiles>[X][Al](Cc1ccccc1OC)C1Cc2ccccc2C1C(OC)OC</smiles>

Esquema 20 
Tabela 1. Reação de 1,2-diidronaftalenos com TTN

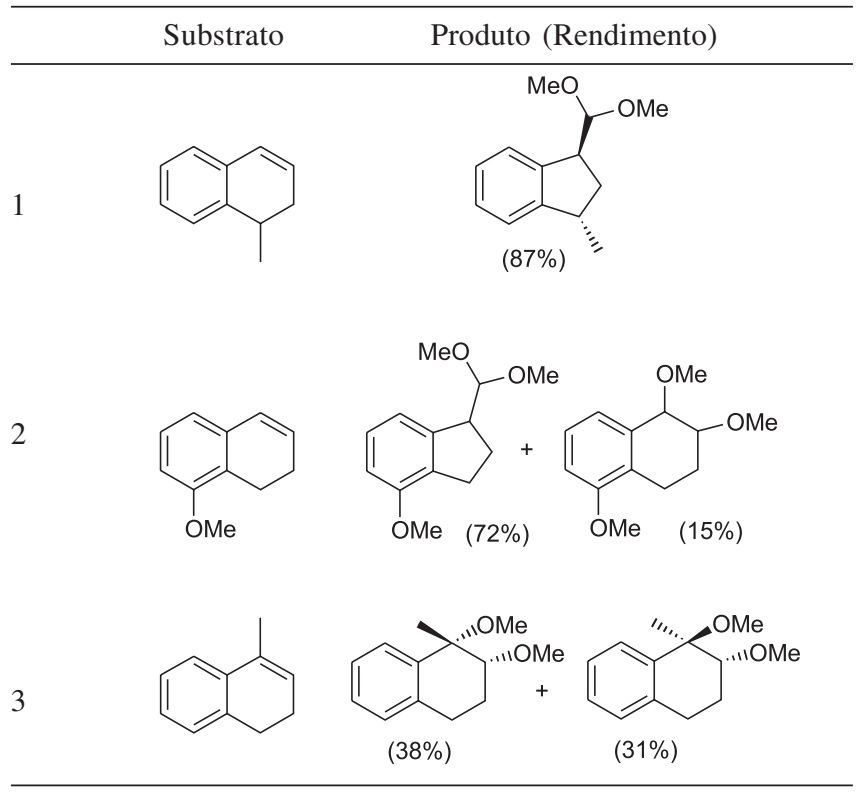

Tabela 2. Contração de 3-alquenóis com TTN em AcOH: $\mathrm{H}_{2} \mathrm{O}$

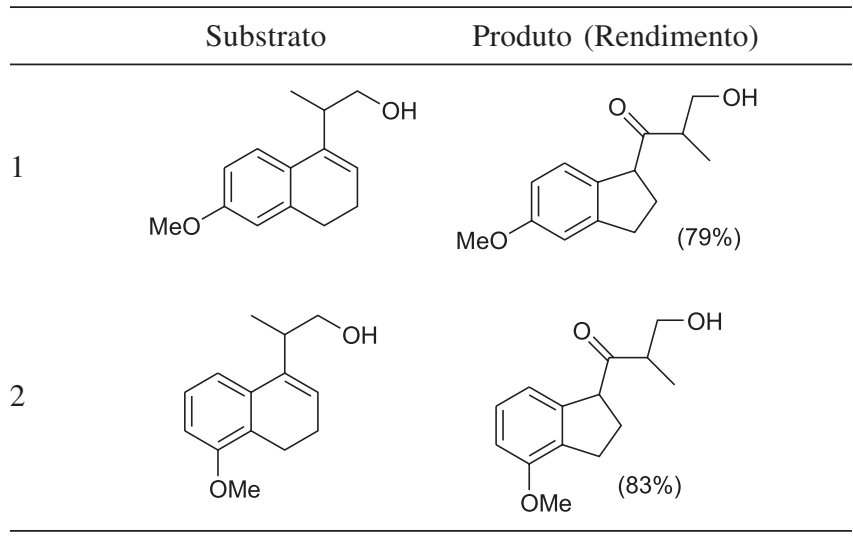

utilizados como substratos nestes rearranjos os correspondentes éteres metílicos e benzílicos ${ }^{30}$ (Esquema 21). O rearranjo de 1,2diidronaftalenos foi recentemente utilizado como etapa chave na síntese do sesquiterpeno fenólico mutisiantol ${ }^{31,32}$ (Esquema 22). Além disso, esta reação foi utilizada na formação do indano em uma recém-desenvolvida abordagem para a síntese diastereosseletiva de 3-fenil-1-indanaminas ${ }^{33}$ (Esquema 23).

A contração de anel de alquenos cíclicos com difluoreto de iodotolueno - um reagente de iodo(III) - foi empregada na formação de indanos a partir de 1,2-diidronaftalenos ${ }^{34}$ (Esquema 24).

\section{REAÇÕES DE CICLIZAÇÃo}

Uma reação de Heck intramolecular da enamida com o iodeto de arila de 33, catalisada por paládio, permitiu obter espiro-indanos, como 34 e 35, em bom rendimento. Esta ciclização foi realizada na presença de um composto de arsênio ou na de acetato de tálio e trifenilfosfina, sendo que estes últimos suprimiram a isomerização da dupla ligação, fornecendo o indano $\mathbf{3 5}^{35}$ (Esquema 25).

Um outro exemplo de ciclização intramolecular catalisada por paládio foi descrito por Crousse et al. ${ }^{36,37}$, que utilizaram o complexo de haloareno-crômio 36 como material de partida para preparação de indanos oticamente ativos. Nos experimentos realiza-

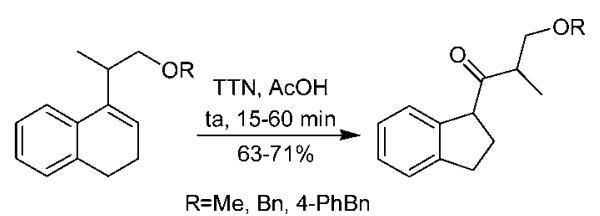

Esquema 21

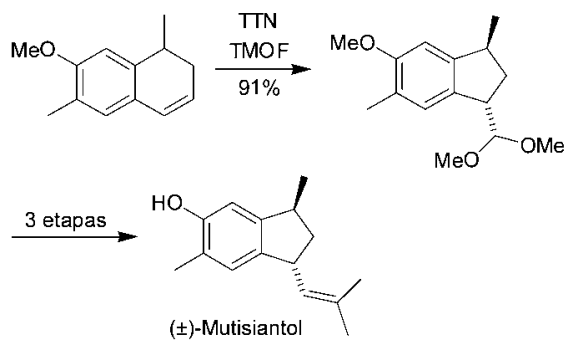

Esquema 22

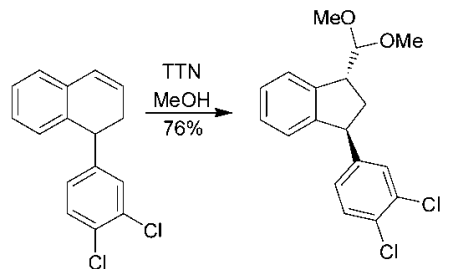<smiles>N[C@H]1C[C@@H](c2ccc(Cl)c(Cl)c2)c2ccccc21</smiles>

Esquema 23

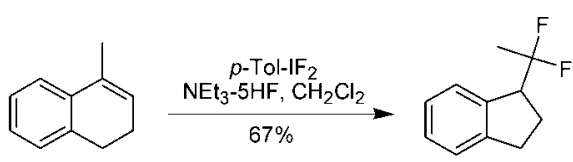

Esquema 24

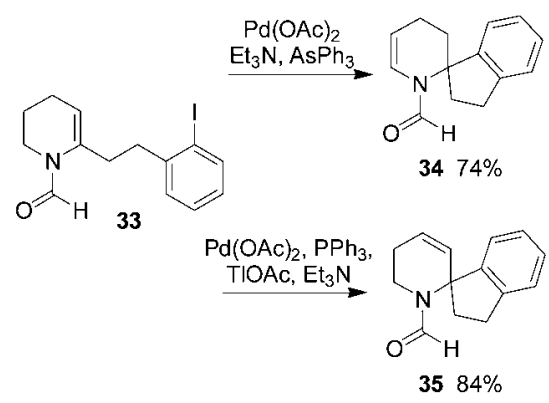

Esquema 25

dos, observou-se que a coordenação com $\mathrm{Cr}(\mathrm{CO})_{3}$ no indano 37 permaneceu inalterada e não houve isomerização ao respectivo indeno (Esquema 26). Realizando-se a ciclização na presença de $\mathrm{CO}$ e metanol comprovou-se a influência dos elementos quirais presentes no complexo 36, o qual forneceu o indano 38 com bom excesso diastereomérico. Esta última foi governada pela quiralidade 
planar de 36 e não pelo centro estereogênico benzílico, como proposto para outras ciclizações. Isto pode ser explicado pelo impedimento estérico ocasionado entre o grupo $\mathrm{Cr}(\mathrm{CO})_{3}$ e um dos ligantes do paládio, no estado de transição proposto para a reação de Heck (Esquema 26). A preparação de um radical arila em um anel coordenado com $\mathrm{Cr}(\mathrm{CO})_{3}$ e seu uso em uma reação de ciclização foi realizada em 2001 pelos mesmos autores ${ }^{37}$. Este procedimento forneceu uma mistura diastereoisomérica dos indanos 40, em bom rendimento, mas sem nenhuma diastereosseletividade, que neste caso não foi influenciada pela quiralidade planar do material de partida nem pelo estereocentro benzílico (Esquema 27).<smiles>C=CCC(OC)c1ccc(C(=O)[O-])cc1Cl</smiles>

$(-)-36$

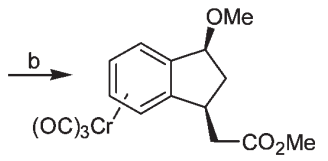

(+)-38 $73 \%,>98 \%$ ed

a: $\mathrm{Pd}(\mathrm{OAc})_{2}, \mathrm{PPh}_{3}, \mathrm{Et}_{3} \mathrm{~N}, \mathrm{MeCN}$

b: $\mathrm{Pd}\left(\mathrm{PPh}_{3}\right)_{4}, \mathrm{Et}_{3} \mathrm{~N}, \mathrm{MeOH}, \mathrm{CO}, \mathrm{MeCN}$, benzeno

Esquema 26

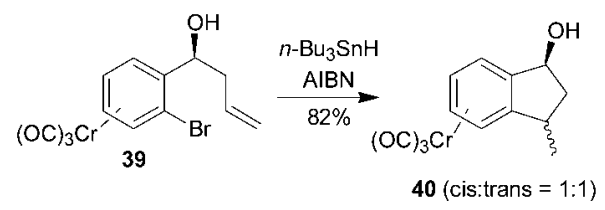

Esquema 27

Uma ciclização carbonilativa catalisada por paládio de diversos orto-iodoestirenos foi utilizada para a síntese de um grande número de 1-indanonas substituídas ${ }^{38}$ (Esquema 28).

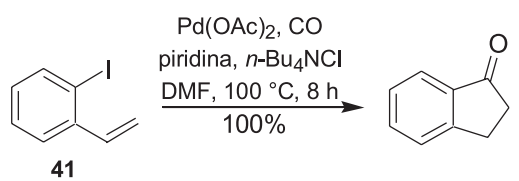

\section{Esquema 28}

Outros metais, além do paládio, também foram utilizados em reações de ciclização visando à síntese de indanos. Catalisadores de níquel, cobre e paládio foram empregados na ciclização de $\omega$ haloesteres para a preparação de 1-indanonas substituídas ${ }^{39}$, como 43 (Esquema 29). Além disso, catalisadores de ródio com ligantes quirais promovem a ciclização enantiosseletiva de iminas aromáti-

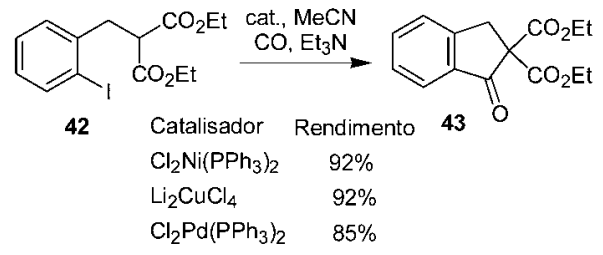

Esquema 29 cas, como 44, o que permite a preparação de uma série de compostos cíclicos, incluindo indanos ${ }^{40}$, como 45 (Esquema 30).

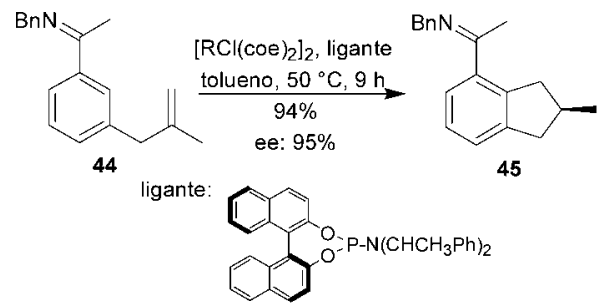

Esquema 30

A ciclização 5-exo-dig do benzino-lítio 49, preparado a partir de 46, forneceu o indano-lítio 50, cuja reação com benzaldeído levou ao indano-4-substituído $\mathbf{5 1} \mathbf{1}^{41}$ (Esquema 31). Uma outra estratégia descrita pelo mesmo grupo para a preparação de indanos envolve a ciclização do organolítio $\mathbf{5 3}$, que forneceu o indano cis-1,3dissubstituído 54a com elevada estereosseletividade. A explicação proposta para esta estereosseletividade baseia-se nos cálculos computacionais realizados para o estado de transição proposto para a reação, indicando que a energia livre de ativação para a ciclização do complexo cis é menor do que para o trans. A alta estereosseletividade obtida no procedimento anterior contrasta com a baixa estereosseletividade da reação radicalar do iodeto $\mathbf{5 2}$ para a preparação do indano 1,3-dissubstituído 54. Contudo, a reação apresentou elevada regiosseletividade na ciclização do radical primário gerado, levando apenas ao anel de cinco membros ${ }^{42}$ (Esquema 32).

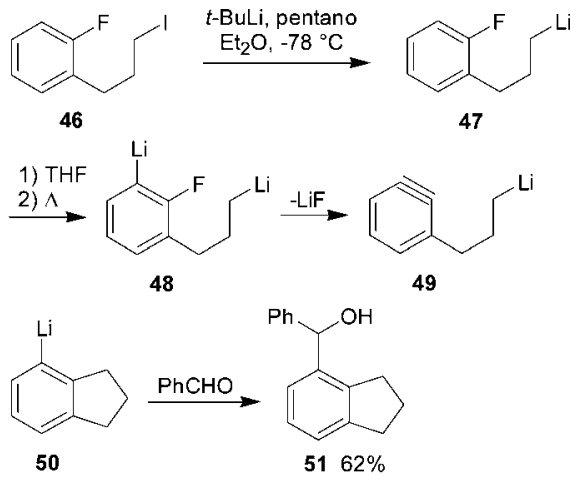

Esquema 31

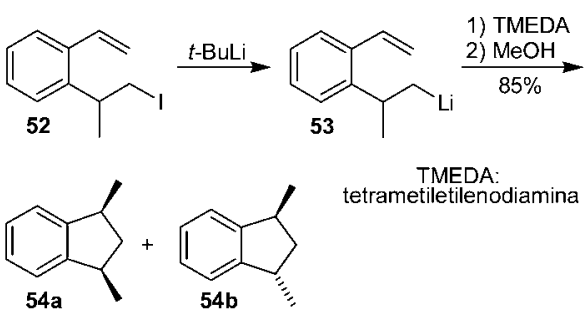

$(12: 1)$

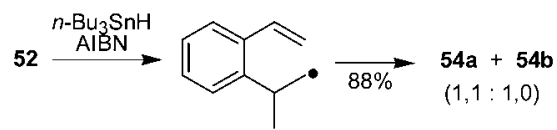

Esquema 32

A metalação do brometo $\mathbf{5 5}$, promovida pela reação com $n$ BuLi, leva a um ataque nucleofílico do carbânion formado à 
carbonila da 1,3-oxazolidina, fornecendo a indanona 56. Esta reação foi empregada na síntese assimétrica da (+)-ribasina, um alcalóide isolado de plantas das famílias Papaveraceae e Fumariaceae $^{43}$ (Esquema 33).

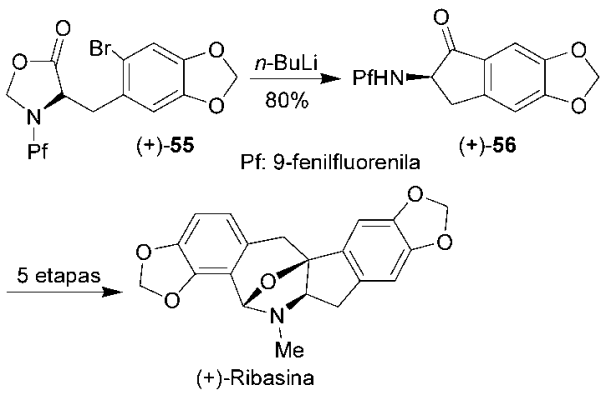

Esquema 33

Um método interessante para a preparação de indanos é a adição nucleofílica intramolecular do anel aromático ao epóxido (composto 57), na presença de $\mathrm{AlCl}_{3}$. $\mathrm{O}$ mecanismo proposto pelos autores mostra a importância do cloreto para formação do produto de ciclização, uma vez que as mesmas condições reacionais empregadas em substratos que não apresentam cloro em sua estrutura forneceram produtos de abertura do epóxido em vez do produto de ciclização $^{44}$ (Esquema 34). Um recente trabalho descreve a obtenção de 2-indanonas catalisada por rutênio a partir de substratos que também contém o grupo funcional epóxido, bem como o de um alquino $^{45}$ (Esquema 35).

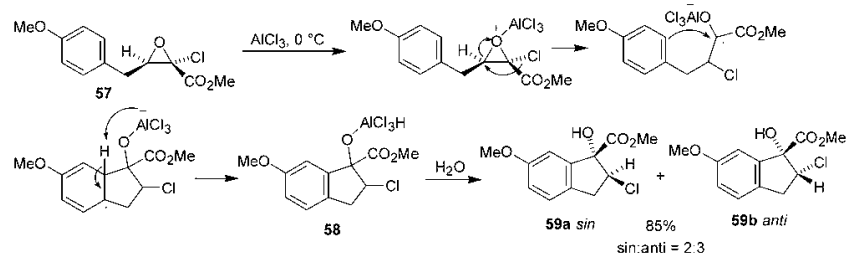

Esquema 34

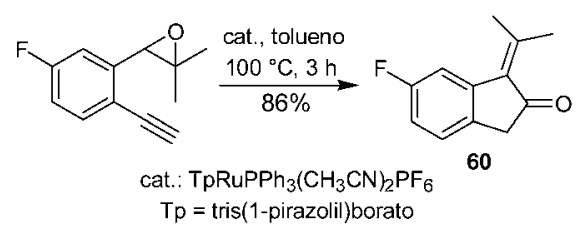

Esquema 35

Uma reação de ciclização do tipo 5-endo-trig, catalisada por paládio, foi empregada na síntese enantiosseletiva dos antagonistas do receptor da endotelina 63 e $\mathbf{6 4}^{46}$ (Esquema 36). A endotelina é um peptídeo que possui atividade vasoconstritora potente dos músculos vasculares lisos e, por isso, acredita-se que a mesma seja responsável por diversas doenças cardiovasculares e pulmonares.

Um outro exemplo de ciclização 5-endo-trig para preparação assimétrica de indanos foi descrito por Déziel et al. $^{47}$. Neste procedimento, a adição eletrofílica do reagente de selênio quiral à olefina 65 forneceu o intermediário seleno-metoxilado 66. O tratamento de 66 com $\mathrm{BF}_{3}$ produziu o selenirânio 67, cuja ciclização levou ao indano 68 (Esquema 37).

A ciclização catalisada por ácido tríflico de derivados do ácido cinâmico é um modo eficiente de preparar 3-fenil-1-indanonas com bom rendimento, como recentemente descrito $^{48}$ (Esquema 38).

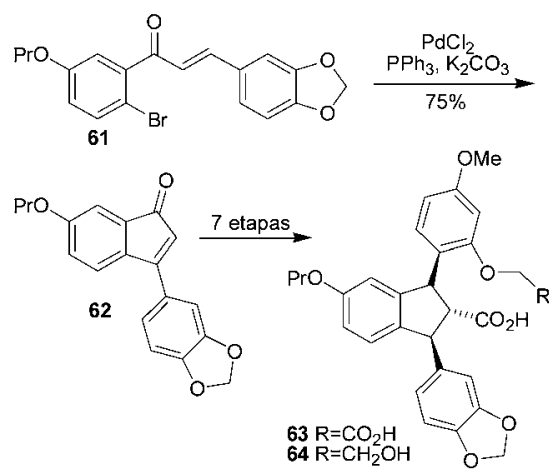

Esquema 36
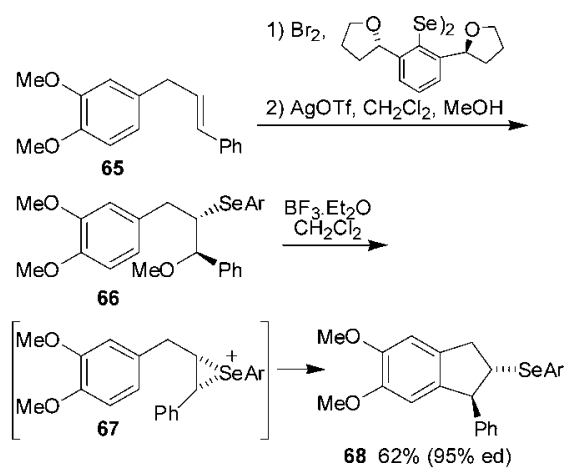

Esquema 37

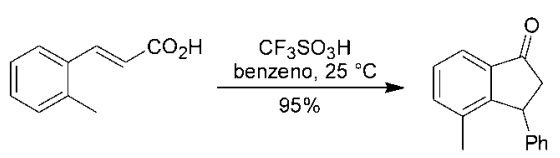

Esquema 38

\section{RESOLUÇÃO DE INDANOS}

Discutiu-se nos parágrafos acima a síntese de indanos oticamente ativos ou não - através de reações em que ocorre a formação de ligações carbono-carbono. Contudo, uma abordagem muito utilizada para a obtenção de indanos oticamente ativos, os quais podem ser utilizados como blocos de construção na síntese de moléculas complexas, é a resolução. Alguns exemplos de resoluções promovidas por enzimas, bem como por catalisadores metálicos, serão discutidos a seguir.

A acetilação enzimática da ( \pm )-3-hidróxi-1-indanona foi estudada por Joly e Nair ${ }^{49}$, mostrando-se de baixa eficiência para duas das três enzimas utilizadas. A enzima Porcine pancreatic lipase (PPL) não produziu a transformação desejada. Nas mesmas condições a Amano PS e a Candida cylindracea lipase (CCL) produziram o álcool desejado, sendo a primeira com maior eficiência (Esquema 39).

A síntese enantiosseletiva da unidade cis-1-amino-2-indanol é de grande utilidade. Nakano et al. ${ }^{50}$ realizaram uma acetilação enzimática do ( \pm -)-cis-1,2-indanodiol usando lipase PS em diferentes solventes, resultando nos monoacetatos 69 e 70 enantiomericamente puros. Cada um destes monoacetatos foi submetido à reação de Ritter, seguida de hidrólise da amida gerada, levando, 
assim, aos dois enantiômeros do cis-1-amino-2-indanol (Esquema 40). Além disso, o cis-1-amino-2-indanol oticamente ativo pode ser preparado através da epoxidação do indeno nas condições de Jacobsen, seguido de reação de Ritter do óxido de indeno forma$\mathrm{do}^{51}$. Uma outra maneira de se obter aminoindanóis oticamente ativos de maneira eficiente foi a resolução do cis- e do trans-1amino-3-indanol utilizando as lipases Pseudomonas cepacia (PSL) e lipase B (CAL-B) ${ }^{52}$.

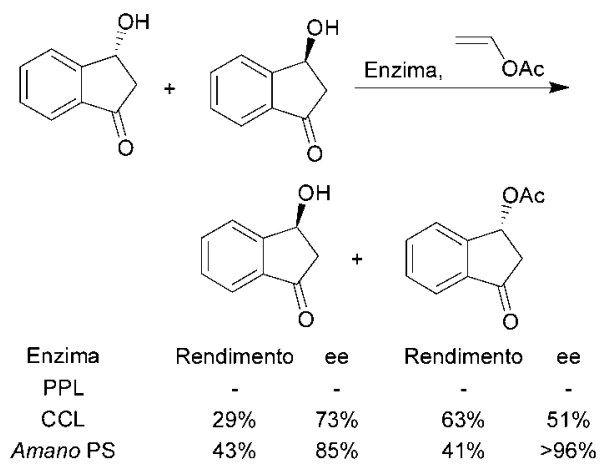

Esquema 39
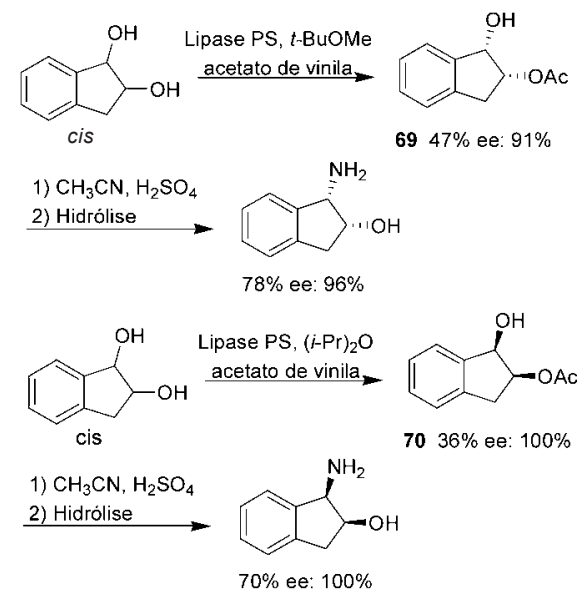

Esquema 40

Conceição et al. ${ }^{53}$ estudaram a redução da 1,2-indanodiona (71) para a obtenção do $(1 S, 2 R)$-cis-1,2-indanodiol, o qual foi submetido à reação de Ritter, formando o $(1 S, 2 R)$-cis-1-amino-2-indanol. Esta redução assimétrica foi realizada com fermento, contendo o microorganismo Trichosporon cutaneum CCT 1903, obtendo-se somente o cis-1,2-indanodiol enantiomericamente puro. É sugerida a formação da benzoína racêmica $\mathbf{7 2}$ por uma redutase não seletiva. Os dois enantiômeros deste composto $(\mathbf{7 4 a} / \mathbf{b})$ estariam em equilíbrio, que seria deslocado pelo consumo do enantiômero $(R)$, através da ação de uma segunda redutase (Esquema 41).

Tarui et al..$^{54}$ fizeram a resolução enzimática do derivado indânico 75, que é um intermediário da síntese de um agonista do receptor da melatonina. O melhor resultado deste estudo foi obtido utilizando a bactéria Bacillus sp. SUI-12, que forneceu o isômero desejado com excesso enantiomérico maior que 95\% (Esquema 42).

Nishibayashi et al. ${ }^{55}$ realizaram uma resolução oxidativa de álcoois racêmicos catalisada por complexos de rutênio quirais. Uma mistura racêmica do 1-indanol foi submetida à reação de transferência de hidrogênio, utilizando-se um complexo de rutênio em quantidades catalíticas e isopropóxido de sódio. Nestas condições a oxidação do $(R)$-1-indanol é 15 vezes mais rápida que a do

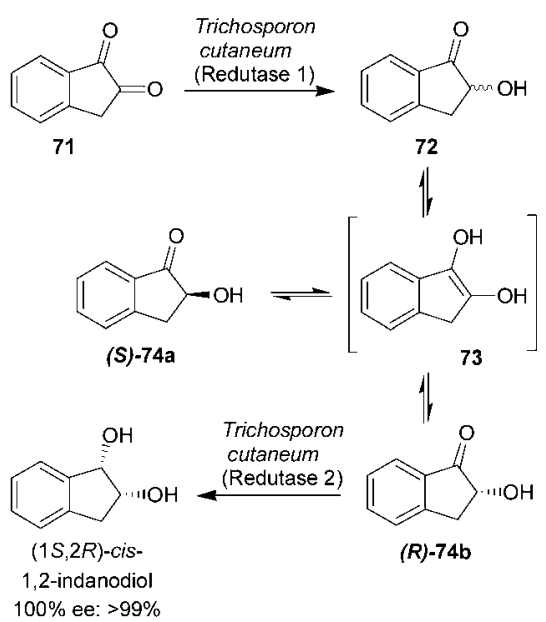

Esquema 41

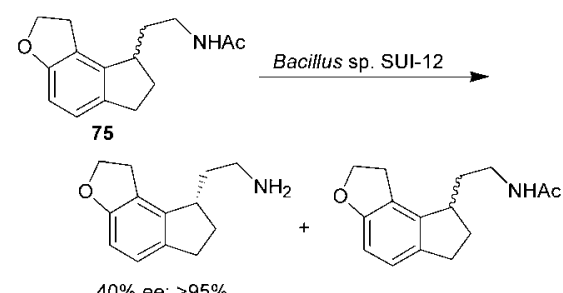

Esquema 42

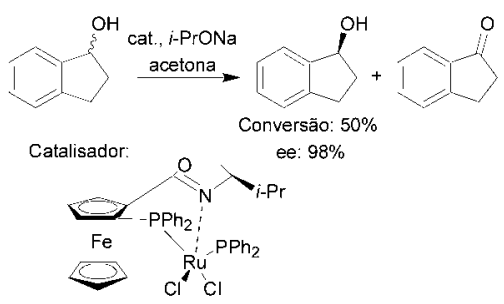

Esquema 43

enantiômero $S$, permitindo a obtenção do $(S)$-1-indanol, com excesso enantiomérico de $98 \%$ (Esquema 43).

Braun e $\operatorname{Kotter}^{56}$ propuseram uma resolução dinâmica de indanóis e seus sililéteres utilizando um complexo quiral de titânio como catalisador. O 1-metil-1-indanol (ou seu silil-derivado) foi tratado com aliltrimetilsilano na presença de quantidades catalílicas do complexo quiral de titânio, sofrendo uma reação de substituição. A forma racêmica dos compostos interage com o complexo quiral gerando dois diastereoisômeros. Eles se relacionam por um rápido equilíbrio com um carbocátion planar aquiral como intermediário. Na reação subsequiente com o aliltrimetilsilano, a forma diastereoisomérica $(S)$ é mais reativa que o outro diastereoisômero formado. Portanto, o alilsilano ataca a face não ocupada pelo complexo de titânio, resultando no isômero observado (Esquema 44).

Hayashi e colaboradores ${ }^{57}$ obtiveram indanos assimétricos pela hidrossililação assimétrica de estirenos catalisada por complexos de paládio. A hidrossililação do estireno $\mathbf{7 6}$ foi realizada com triclorossilano e com um catalisador, que foi gerado in situ misturando $\left[\mathrm{PdCl}\left(\pi-\mathrm{C}_{3} \mathrm{H}_{5}\right)\right]_{2}$ e uma fosfina quiral. Foram obtidos os produtos de ciclização 77 e de adição $\mathbf{7 8}$ e $\mathbf{7 9}$, em $74 \%$ de rendimento global, nas proporções de 47:42:21, respectivamente. O trans-1- 


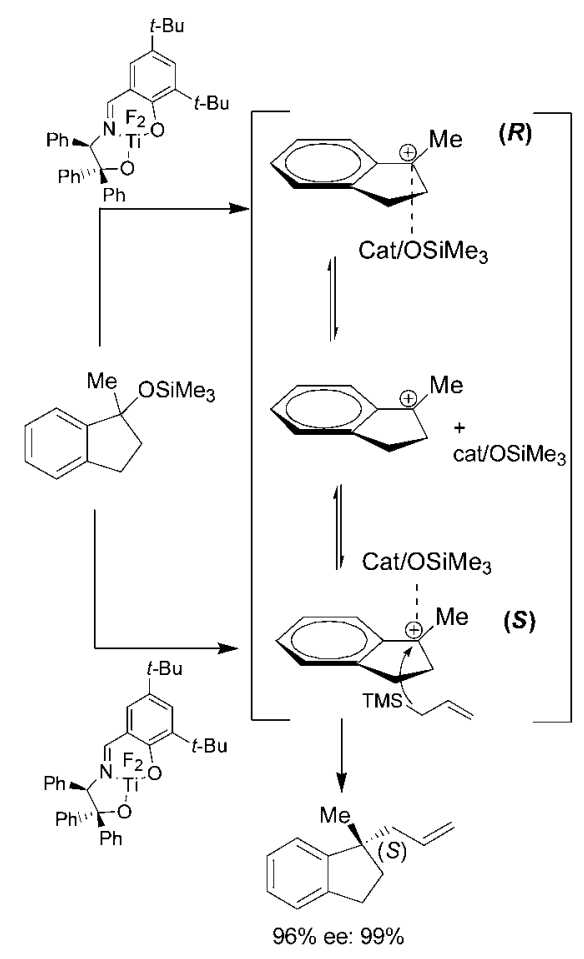

Esquema 44

metil-2-(triclorossililmetil)indano $\mathbf{7 7}$ foi oxidado, primeiramente por peróxido de hidrogênio e, posteriormente, por PCC. Após as oxidações, o produto foi submetido a uma desformilação com quantidades estequiométricas de $\mathrm{RhCl}\left(\mathrm{PPh}_{3}\right)$, gerando o $(S)$-1metilindano 81 (Esquema 45).
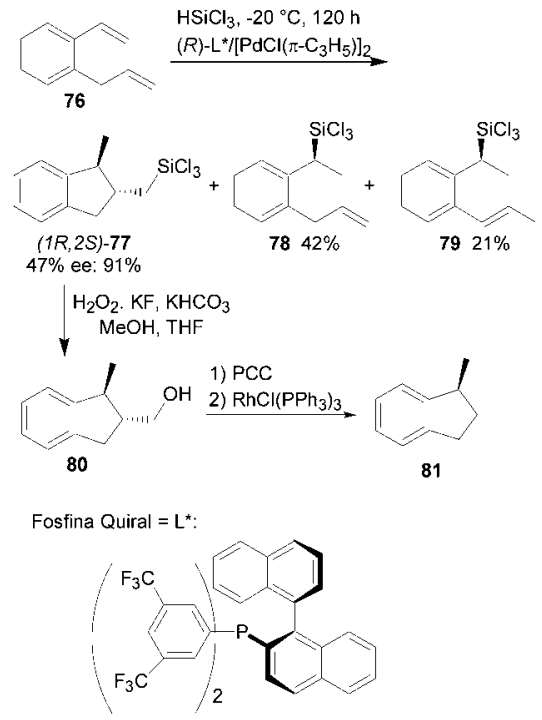

Esquema 45

A proposta mecanística para a seletividade está mostrada no Esquema 46. O complexo quiral de paládio liga-se à dupla ligação, havendo equilíbrio entre as formas 82 e 83 . O diastereoisômero 83 é muito mais reativo que o $\mathbf{8 2}$ frente à eliminação redutiva, que gera o composto 78 e seu isômero 79. Já o diastereoisômero 82 gera o composto ciclizado 77, pela inserção do carbono 2 do substituinte propenil no carbono contendo o paládio.

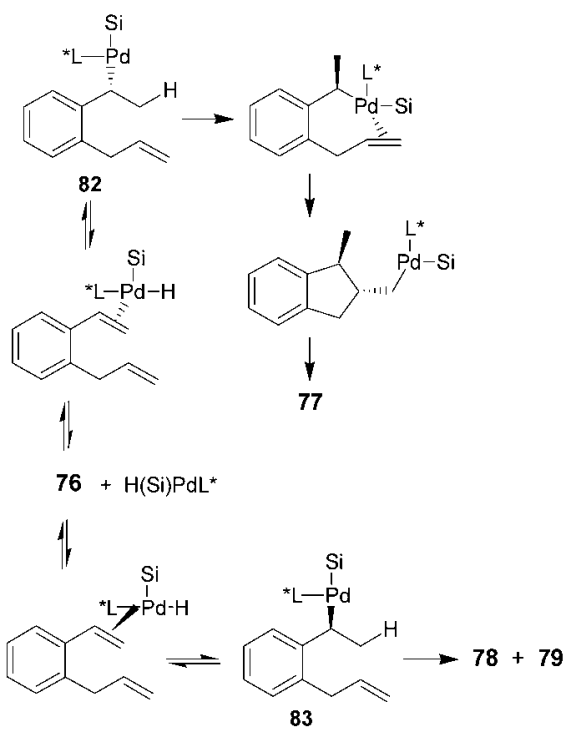

Esquema 46

\section{CONCLUSÃO}

A síntese de indanos pode ser realizada de maneira eficiente por uma variedade de métodos, destacando-se: i) a reação de acilação intramolecular de Friedel-Crafts que pode ser utilizada na síntese de indanonas funcionalizadas; ii) as reações de cicloadição catalisadas por metais de transição; iii) as reações de contração de anel promovidas por tálio(III) e iodo(III); iv) reações de ciclização que podem ser realizadas por diversos reagentes e v) a resolução de indanos. Esperamos que os métodos aqui descritos possam servir de inspiração para o surgimento de novas abordagens de síntese de indanos.

\section{AGRADECIMENTOS}

Os autores agradecem à FAPESP, ao CNPq e à CAPES pelo contínuo apoio financeiro.

\section{REFERÊNCIAS}

1. Dorsey, B. D.; Levin, R. B.; McDaniel, S. L.; Vacca, J. P.; Guare, J. P.; Darke, P. L.; Zugay, J. A.; Emini, E. A.; Schleif, W. A.; Quintero, J. C.; Lin, J. H.; Chen, I.-W.; Holloway, M. K.; Fitzgerald, P. M. D.; Axel, M. G.; Ostovic, D.; Anderson, P. S.; Huff, J. R.; J. Med. Chem. 1994, 37, 3443.

2. Ghosh, A. K.; Bilcer, G.; Schiltz, G.; Synthesis 2001, 2203.

3. Sugimoto, H.; Iimura, Y.; Yamanishi, Y.; Yamatsu, K.; J. Med. Chem. 1995, 38,4821 .

4. Sugimoto, H.; Pure Appl. Chem. 1999, 71, 2031.

5. Sprecker, M. A.; Weiss, R. A.; Belko, R. P.; Molner, E. A.; International Flavors \& Fragances Inc. US 6.342.612 B1: EUA, 2002.

6. Sengupta, S.; Mondal, S.; Tetrahedron Lett. 1999, 40, 3469.

7. Hanessian, S.; Ma, J.; Tetrahedron Lett. 2001, 42, 8785.

8. Wu, C.; Nakamura, H.; Murai, A.; Inouye, S.; Tetrahedron 2001, 57, 9575.

9. Bogeso, K. P.; Christensen, A. V.; Hyttel, J.; Liljefors, T.; J. Med. Chem. 1985, 28, 1817.

10. Davies, H. M. L.; Gregg, T. M.; Tetrahedron Lett. 2002, 43, 4951.

11. Ho, T.-L.; Lee, K.-Y.; Chen, C.-K.; J. Org. Chem. 1997, 62, 3365

12. Fillion, E.; Fishlock, D.; Org. Lett. 2003, 5, 4653.

13. Cui, D.-M.; Zhang, C.; Kawamura, M.; Shimada, S.; Tetrahedron Lett. 2004, 45, 1741 .

14. Katrizky, A. R.; Zhang, G.; Xie, L.; Synth. Commun. 1997, 27, 2467.

15. Kim, S.; Kitano, Y.; Tada, M.; Chiba, K.; Tetrahedron Lett. 2000, 41, 7079.

16. Bradley, A. Z.; Johnson, R. P.; J. Am. Chem. Soc. 1997, 119, 9917.

17. Kotha, S.; Sreenivasachary, N.; Brahmachary, E.; Tetrahedron Lett. 1998, 39, 2805.

18. Kotha, S.; Brahmachary, E.; J. Org. Chem. 2000, 65, 1359.

19. Grigg, R.; Sridharan, V.; Zhang, J.; Tetrahedron Lett. 1999, 40, 8277.

20. Yamamoto, Y.; Nagata, A.; Itoh, K.; Tetrahedron Lett. 1999, 40, 5035. 
21. Jeevanandam, A.; Korivi, R. P.; Huang, I.-w.; Cheng, C.-H.; Org. Lett. 2002, 4, 807.

22. Dalton, A. M.; Zhang, Y.; Davie, C. P.; Danheiser, R. L.; Org. Lett. 2002, 4, 2465.

23. Hashimi, A. S. K.; Ding, L.; Bats, J. W.; Fischer, P.; Frey, W.; Chem. Eur. J. 2003, 9, 4339

24. Shibata, T.; Fujimoto, T.; Yokota, K.; Takagi, K.; J. Am. Chem. Soc. 2004, $126,8382$.

25. Silva Jr, L. F.; Tetrahedron 2002, 58, 9137.

26. Ferraz, H. M. C.; Silva, L. F., Jr.; Aguilar, A. M.; Vieira, T. O.; J. Braz. Chem. Soc. 2001, 12, 680.

27. Ferraz, H. M. C.; Silva , L. F., Jr; Vieira, T. O.; Tetrahedron 2001, 57, 1709.

28. Ferraz, H. M. C.; Santos, A. P.; Silva , L. F., Jr; Vieira, T. O.; Synth. Commun. 2000, 30, 751 .

29. Ferraz, H. M. C.; Silva, L. F., Jr; Tetrahedron 2001, 57, 9939.

30. Ferraz, H. M. C.; Silva, L. F., Jr.; Synthesis 2002, 1033.

31. Ferraz, H. M. C.; Aguilar, A. M.; Silva, L. F., Jr.; Synthesis 2003, 1031

32. Ferraz, H. M. C.; Aguilar, A. M.; Silva, L. F., Jr; Tetrahedron 2003, 59, 5817.

33. Silva, L. F., Jr.; Siqueira, F. A.; resultados não publicados.

34. Hara, S.; Nakahigashi, J.; Ishi-i, K.; Fukuhara, T.; Yoneda, N.; Tetrahedron Lett. 1998, 39, 2589.

35. Ripa, L.; Hallberg, A.; J. Org. Chem. 1997, 62, 595.

36. Crousse, B.; Xu, L.-H.; Bernardinelli, G.; Kündig, E. P.; Synlett 1998, 658.

37. Kündig, E. P.; Ratni, H.; Crousse, B.; Bernardinelli, G.; J. Org. Chem. 2001, 66, 1852.

38. Gagnier, S. V.; Larock, R. C.; J. Am. Chem. Soc. 2003, 125, 4804.

39. Negishi, E.; Makabe, H.; Shimoyama, I.; Wu, G.; Zhang, Y.; Tetrahedron 1998, 54, 1095.
40. Thalji, R. K.; Ellman, J. A.; Bergman, R. G.; J. Am Chem. Soc. 2004, 126, 7192.

41. Bailey, W. F.; Longstaff, S. C.; J. Org. Chem. 1998, 63, 432.

42. Bailey, W. F.; Mealy, M. J.; Wiberg, K. B.; Org. Lett. 2002, 4, 791.

43. Ollero, L.; Castedo, L.; Domínguez, D.; Tetrahedron 1999, 55, 4445.

44. Lin, J.-R.; Gubaidullin, A. T.; Mamedov, V. A.; Tsuboi, S.; Tetrahedron 2003, 59, 1781.

45. Madhushaw, R. J.; Lin, M.-Y.; Sohel, S. M. A.; Liu, R.-S.; J. Am. Chem. Soc. 2004, 126, 6895.

46. Clark, W. M.; Tickner-Eldridge, A. M.; Huang, G. K.; Pridgen, L. N.; Olsen, M. A.; Mills, R. J.; Lantos, I.; Baine, N. H.; J. Am. Chem. Soc. 1998, $120,4550$.

47. Déziel, R.; Malenfant, E.; Thibault, C.; Tetrahedron Lett. 1998, 39, 5493.

48. Rendy, R.; Zhang, Y.; McElrea, A.; Gomez, A.; Klump, D. A.; J. Org. Chem. 2004, 69, 2340.

49. Joly, S.; Nair, M. S.; Tetrahedron: Asymmetry 2001, 12, 2283.

50. Nakano, S.; Igarashi, Y.; Nohira, H.; Tetrahedron: Asymmetry 2001, 12, 59.

51. Kobayashi, T.; Tanaka, K.; Miwa, J.; Katsumura, S.; Tetrahedron: Asymmetry 2004, 15, 185.

52. López-Garcia, M.; Alfonso, I.; Gotor, V.; Chem. Eur. J. 2004, 10, 3006

53. Conceição, G. J. A.; Moran, P. J. S.; Rodrigues, J. A. R.; Tetrahedron: Asymmetry 2003, 14, 2327.

54. Tarui, N.; Nagano, Y.; Sakane, T.; Matsumoto, K.; Kawada, M.; Uchikawa, O.; Ohkawa, S.; Nakahama, K.; J. Bioscience Bioeng. 2002, 93, 44.

55. Nishibayashi, Y.; Yamauchi, A.; Onodera, G.; Uemura, S.; J. Org. Chem. 2003, 68, 5875

56. Braun, M.; Kotter, W.; Angew. Chem. Int. Ed. 2004, 43, 514.

57. Hayashi, T.; Hirate, S.; Kitayama, K.; Tsuji, H.; Torii, A.; Uozumi, Y.; J. Org. Chem. 2001, 66, 1441. 